\title{
Tratamento medicamentoso da migrânea vestibular
}

\author{
Drug treatment of vestibular migraine \\ Tratamiento medicamentoso de la migraña vestibular
}

Recebido: 21/06/2021 | Revisado: 27/06/2021 | Aceito: 28/06/2021 | Publicado: 13/07/2021

\author{
Siméia de Castro Ramos \\ ORCID: https://orcid.org/0000-0003-2401-8347 \\ Universidade Federal do Maranhão, Brasil \\ E-mail: simeia.ramos@hotmail.com \\ Sueli de Souza Costa \\ ORCID: https://orcid.org/0000-0003-4127-7324 \\ Universidade Federal do Maranhão, Brasil \\ E-mail: scsueli@gmail.com \\ Patrick Rademaker Burke \\ ORCID: https://orcid.org/0000-0001-7114-2901 \\ Universidade Federal do Maranhão, Brasil \\ E-mail: patrickotoneuro@gmail.com
}

\begin{abstract}
Resumo
Migrânea vestibular corresponde a um dos mais frequentes diagnósticos em otoneurologia. É considerada a causa mais comum de distúrbios de equilíbrio. Seu diagnóstico é baseado em sintomas vestibulares recorrentes, história de migrânea e a associação dos sintomas migranosos com os sintomas vestibulares em um período que varia de 5 minutos a 72 horas. $\mathrm{O}$ tratamento medicamentoso da migrânea vestibular objetiva o controle dos sintomas do paciente na fase aguda, bem como na profilaxia das eventuais crises. O presente trabalho é uma revisão integrativa da literatura, cujo objetivo é identificar e descrever o tratamento medicamentoso da migrânea vestibular, a fim de controlar os sintomas do paciente na fase aguda e na profilaxia das eventuais crises. A busca foi realizada nas bases de dados eletrônicas SCIELO, PUBMED e LILACS, incluindo trabalhos publicados entre 2016 e 2021, em língua portuguesa e inglesa. A seleção dos estudos, extração de dados e validação foram realizadas de forma independente por dois autores, obtendo amostra de 14 artigos. Todos os dados da pesquisa foram analisados no Microsoft Excel e posteriormente os resultados foram transformados em tabelas para melhor interpretação das informações coletadas. Constatou-se, através da análise dos 14 artigos, que 7 (50\%) estudos utilizaram tratamento com antidepressivos, betabloqueadores e anticonvulsivantes concomitantemente; 9 (64\%) utilizaram propranolol com melhora dos sintomas; 2 (14\%) utilizaram a toxina botulínica e em apenas 1 (7\%) foi utilizado a injeção com gentamicina. Assim, foi possível estruturar de forma sistemática os medicamentos mais utilizados na profilaxia e na crise da migrânea vestibular, lançando base para escolha mais adequada do fármaco.
\end{abstract}

Palavras-chave: Transtornos de enxaqueca; Doenças vestibulares; Tratamento farmacológico.

\begin{abstract}
Vestibular migraine corresponds to one of the most frequent diagnoses in otoneurology. It is considered the most common cause of balance disorders. Its diagnosis is based on recurrent vestibular symptoms, history of migraine and association of migraine symptoms with vestibular symptoms over a period ranging from 5 minutes to 72 hours. The drug treatment of vestibular migraine aims to control the patient's symptoms in the acute phase, as well as in the prophylaxis of eventual crises. The present work is an integrative literature review, whose objective is to identify and describe the drug treatment of vestibular migraine, in order to control the patient's symptoms in the acute phase and in the prophylaxis of possible crises. The search was carried out in the electronic databases SCIELO, PUBMED and LILACS, including works published between 2016 and 2021, in Portuguese and English. The selection of studies, data extraction and validation were carried out independently by two authors, obtaining a sample of 14 articles. All research data were analyzed in Microsoft Excel and later the results were transformed into tables for a better interpretation of the information collected. It was found, through the analysis of the 14 articles, that $7(50 \%)$ studies used treatment with antidepressants, beta-blockers and anticonvulsants concomitantly; 9 (64\%) used propranolol with symptom improvement; 2 (14\%) used botulinum toxin and only 1 (7\%) used gentamicin injection. Thus, it was possible to systematically structure the drugs most used in prophylaxis and in the vestibular migraine crisis, laying the basis for a more adequate choice of the drug.
\end{abstract}

Keywords: Migraine disorders; Vestibular diseases; Drug therapy. 


\begin{abstract}
Resumen
La migraña vestibular corresponde a uno de los diagnósticos más frecuentes en otoneurología. Se considera la causa más común de trastornos del equilibrio. Su diagnóstico se basa en síntomas vestibulares recurrentes, antecedentes de migraña y la asociación de síntomas de migraña con síntomas vestibulares durante un período que va de 5 minutos a 72 horas. El tratamiento farmacológico de la migraña vestibular tiene como objetivo controlar los síntomas del paciente en la fase aguda, así como en la profilaxis de eventuales crisis. El presente trabajo es una revisión integradora de la literatura, cuyo objetivo es identificar y describir el tratamiento farmacológico de la migraña vestibular, con el fin de controlar los síntomas del paciente en la fase aguda y en la profilaxis de posibles crisis. La búsqueda se realizó en las bases de datos electrónicas SCIELO, PUBMED y LILACS, incluyendo trabajos publicados entre 2016 y 2021 , en portugués e inglés. La selección de estudios, extracción de datos y validación fueron realizadas de forma independiente por dos autores, obteniendo una muestra de 14 artículos. Todos los datos de la investigación fueron analizados en Microsoft Excel y posteriormente los resultados se transformaron en tablas para una mejor interpretación de la información recolectada. Se encontró, mediante el análisis de los 14 artículos, que 7 (50\%) estudios utilizaron tratamiento con antidepresivos, betabloqueantes y anticonvulsivos de forma concomitante; 9 (64\%) usaron propranolol con mejoría de los síntomas; 2 (14\%) usó toxina botulínica y solo 1 (7\%) usó la inyección de gentamicina. Así, fue posible estructurar sistemáticamente los fármacos más utilizados en profilaxis y en la crisis migrañosa vestibular, sentando las bases para una elección más adecuada del fármaco.
\end{abstract}

Palabras clave: Trastornos de migraña; Enfermedades vestibulares; Tratamiento farmacológico.

\title{
1. Introdução
}

A migrânea vestibular (MV) é uma das causas neurológicas mais comuns de vertigem em adultos, com uma prevalência entre $1 \%$ e 2,7\% na população em geral. Afeta mais mulheres em uma faixa etária em torno de 40 anos de idade (Beh, 2019).

A fisiopatologia da MV ainda não está totalmente esclarecida. No entanto, acredita-se que há estímulos noradrenérgicos e serotoninérgicos ativando os núcleos vestibulares durante as crises de migrânea causando sintomas vestibulares. Pelo fato de haver conexão entre o núcleo trigeminal caudal e os núcleos vestibulares, pode-se causar uma inflamação neurogênica do sistema trigeminal e consequente hiperexcitabilidade vestibular, ocasionando sintomas como: dor latejante, nistagmo espontâneo, hipersensibilidade à luz e ao som, dentre outros (Barbosa \& Villa, 2016; Lapira, 2019; Huang, Wang, \& Kheradmand, 2020).

Os critérios diagnósticos para migrânea vestibular foram incluídos em um apêndice na terceira edição da Classificação Internacional de Cefaleias em 2013 pela Bárány Society em conjunto com a Internacional Headache Society. Para que haja o diagnóstico de migrânea vestibular, o paciente tem que apresentar 5 ou mais episódios de sintomas vestibulares de intensidade moderada a severa, com duração de 5 minutos a 72 horas. O paciente também deve ter história prévia ou atual de migrânea, com ou sem aura e apresentar uma ou mais características de migrânea, em pelo menos 50\% dos episódios vestibulares. A cefaleia deve ter pelo menos duas características a seguir: unilateral, pulsátil, intensidade moderada ou severa, agravada por atividade física rotineira. O paciente também deve apresentar fotofobia, fonofobia e aura visual que não seja explicado por outro diagnóstico vestibular ou de cefaleia (Lempert, 2013).

Há também os critérios para que o paciente seja diagnosticado como portador de uma provável migrânea vestibular, ou seja, este paciente deve apresentar no mínimo 5 episódios de sintomas vestibulares, com mesma duração e intensidade descritos anteriormente para MV, mas com um diferencial de que pode apresentar ou não história prévia ou atual de migrânea ou ter apenas a cefaleia, que pode ser unilateral, pulsátil, de moderada a severa intensidade, que se agrava com atividade física, bem como apresentar fotofobia, fonofobia e aura visual, não devendo ser melhor explicado por outro diagnóstico (Lempert, 2013).

Durante as crises de migrânea vestibular os pacientes podem apresentar cefaleia, vertigem, zumbido, hiporreflexia e hiperreflexia vestibular. No intervalo entre as crises, tanto o exame físico como as avaliações auditivas e vestibulares geralmente não têm anormalidades, sendo observado somente a hiperreflexia labiríntica como alteração vestibular (Morganti et al., 2016). 
O tratamento medicamentoso da migrânea vestibular objetiva o controle dos sintomas do paciente na fase aguda, bem como na profilaxia das eventuais crises e consiste no uso de antidepressivos, anticonvulsivantes, anti-hipertensivos, dentre outros (Salmito et al., 2020).

Diante da contextualização acima, justifica-se este trabalho pelo fato da migrânea vestibular ser um dos mais frequentes diagnósticos em otoneurologia (Morganti et al., 2016). Por ser, ainda, clinicamente subdiagnosticada e ter um alto impacto nos custos com a saúde (Huang et al., 2020). Seu tratamento tem fundamental importância por ter um impacto na vida diária e no bem-estar dos pacientes (Barbosa \& Villa, 2016).

Existem poucos estudos sobre o tema, sendo o mais recente o artigo publicado em 2021, que relata sobre os fatores que influenciam na resposta ao tratamento medicamentoso da migrânea vestibular (Dornhoffer, Liu, Donaldson, \& Rizk, 2021). Desta forma, este estudo visou identificar e descrever o tratamento medicamentoso da migrânea vestibular, a fim de controlar os sintomas do paciente na fase aguda e na profilaxia das eventuais crises, adequando o tratamento ideal para cada paciente de acordo com sua especificidade, comorbidades e sintomas.

\section{Metodologia}

O presente trabalho consiste em uma revisão integrativa da literatura, pois sintetiza resultados sobre um tema de maneira ordenada e sistemática (Ercole, Melo, \& Alcoforado, 2014). Inicialmente houve a elaboração do principal conceito norteador da pesquisa: “Quais os tratamentos medicamentosos mais utilizados para a migrânea vestibular?”, após realizou-se a busca de trabalhos relacionados ao tema publicados nos anos de 2016 a 2021, sempre em concordância aos critérios de inclusão e de exclusão. Em seguida, iniciou-se a coleta de dados e, posteriormente, a análise crítica dos artigos relevantes, concluindo-se com a apresentação e discussão dos resultados.

A busca foi realizada nas bases de dados eletrônicas Scientific Electronic Library Online (SCIELO), Publicações Médicas (PUBMED) e Literatura Latino-Americana do Caribe em Ciências da Saúde (LILACS). As referências que preencheram os critérios de inclusão foram avaliadas com relação ao conteúdo publicado e estrutura do trabalho. A seleção dos descritores foi feita mediante consulta aos Descritores de Assunto em Ciências da Saúde (DECs). Os seguintes descritores em língua portuguesa e inglesa foram considerados: "transtornos de enxaqueca" (descritor 1); "doenças vestibulares" (descritor 2), "tratamento farmacológico" (descritor 3); "migraine disorders" (descritor 4); "vestibular diseases" (descritor 5) e "drug therapy" (descritor 6).

Para a seleção dos estudos foram definidos os critérios de inclusão e exclusão. Assim, os critérios de inclusão dos artigos foram as obras em língua portuguesa e inglesa que abordaram temas relacionados à migrânea vestibular, com ideias claras, objetivas e condizentes com o estudo. Por sua vez, foram excluídos: publicações que não se referiram à migrânea vestibular, as obras que não estavam publicadas na íntegra, os trabalhos em língua distintas da portuguesa e inglesa, bem como os artigos em duplicidade.

Foram utilizados etapas e descritores em categorias para a seleção dos artigos nas bases de dados, conforme a seguir: 1) LILACS: a) "transtornos de enxaqueca" e "doenças vestibulares"; b) "transtornos de enxaqueca" e "tratamento farmacológico"; c) "doenças vestibulares" e "tratamento farmacológico"; d) "transtornos de enxaqueca" e "doenças vestibulares" e "tratamento farmacológico; e) "migraine disorders" and "vestibular diseases"; f) "migraine disorders" and "drug therapy"; g) "vestibular diseases" and "drug therapy"; h) "migraine disorders" and "vestibular diseases" and "drug therapy"; 2) PUBMED: a) "migraine disorders" and "vestibular diseases"; b) "migraine disorders" and "drug therapy"; c) "vestibular diseases" and "drug therapy"; d) "migraine disorders" and "vestibular diseases" and "drug therapy", sendo feito rodada diferente em virtude da ausência de resultados na língua portuguesa; e 3) SCIELO: a) "transtornos de enxaqueca" e 
"tratamento farmacológico"; b) "migraine disorders" and "drug therapy"; c) "vestibular diseases" and "drug therapy", sendo feito rodada diferente em virtude da ausência de resultados com o esquematizado para as bases anteriores.

Para a avaliação dos artigos selecionados, elaborou-se um instrumento sistemático baseado no diagrama flow (Moher, 2009) para a coleta das informações de forma independente por dois autores, visando responder à questão norteadora do estudo, no qual os dados relevantes foram dispostos em uma tabela contendo o ano do estudo, autor, título, tipo de estudo, classe de medicamento, medicamentos utilizados e resultado dos autores. As discrepâncias existentes na escolha dos artigos foram discutidas entre os autores do presente trabalho.

Os dados obtidos foram analisados de forma sistemática através da ordenação, classificação, análise final e tabulação dos artigos. Por se tratar de uma pesquisa bibliográfica, este estudo não necessitou de aprovação do Comitê de Ética em Pesquisa, fundamentando-se nas diretrizes e normas regulamentadoras estabelecidas nas resoluções n 466/2012 e 580/2018 do Conselho Nacional de Saúde do Ministério da Saúde.

\section{Resultados}

A combinação entre os descritores no processo de busca resultou, inicialmente, em 1897 artigos. Os achados estão estabelecidos na Tabela 1.

Tabela 1. Disposição do número de artigos conforme arranjos dos descritores utilizados na pesquisa em base de dados.

\begin{tabular}{|c|c|c|c|}
\hline COMBINAÇÃO DE DESCRITORES & LILACS & PUBMED & SCIELO \\
\hline $1 \mathrm{E} 2$ & 6 & - & - \\
\hline $1 \mathrm{E} 3$ & 4 & - & 1 \\
\hline $2 \mathrm{E} 3$ & 6 & - & - \\
\hline $1 \mathrm{E} 2 \mathrm{E} 3$ & 1 & - & - \\
\hline 4 AND 5 & 6 & 191 & - \\
\hline 4 AND 6 & 8 & 1388 & 2 \\
\hline 5 AND 6 & 10 & 251 & 1 \\
\hline 4 AND 5 AND 6 & 1 & 21 & - \\
\hline Total & 42 & 1851 & 4 \\
\hline
\end{tabular}

Legenda: Transtornos de enxaqueca (descritor 1), Doenças vestibulares (descritor 2), Tratamento farmacológico (descritor 3), Migraine disorders (descritor 4), Vestibular diseases (descritor 5), Drug therapy (descritor 6).

Fonte: Autores. Dados obtidos nas bases de dados LILACS, PUBMED e SCIELO.

Após a leitura e análise dos artigos encontrados, eliminando-se a duplicidade dos mesmos, atendendo aos critérios de inclusão e de exclusão e a análise crítica, obteve-se uma amostra final de 14 publicações que atenderam ao conceito norteador do estudo (Figura 1). O percentual de resultados das publicações por ano nas bases de dados LILACS, PUBMED e SCIELO da amostra final (Quadro 1). Todos os dados relevantes do trabalho foram sintetizados em um quadro para melhor visualização da catalogação (Quadro 2). 
Figura 1. Processo de busca e análise dos trabalhos, baseado no diagrama flow de revisão PRISMA 2009 (Moher et al., 2009).

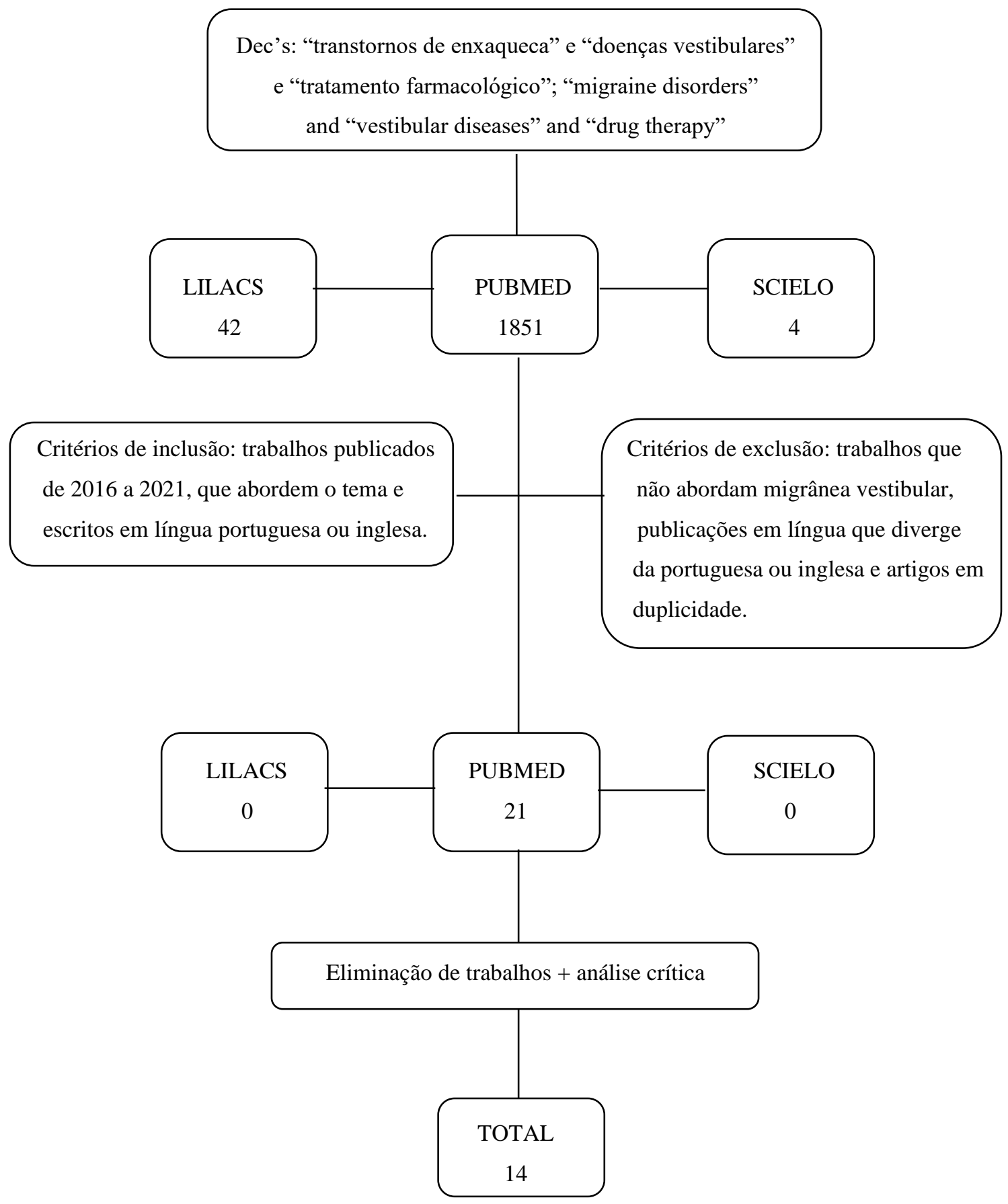

Fonte: Autores.

Quadro 1. Percentual de resultados das publicações por ano da amostra final nas bases de dados do estudo.

\begin{tabular}{|c|c|c|c|c|c|}
\hline 2016 & 2017 & 2018 & 2019 & 2020 & 2021 \\
\hline $28,5 \%$ & $21,4 \%$ & - & $14,2 \%$ & $28,5 \%$ & $7,1 \%$ \\
\hline
\end{tabular}

Fonte: Autores. 
Quadro 2. Síntese dos estudos publicados sobre o tratamento medicamentoso da migrânea vestibular.

\begin{tabular}{|c|c|c|c|c|c|}
\hline Autor & Título & Tipo de Estudo & Classe de Medicamento & Medicamentos & Resultado dos Autores \\
\hline $\begin{array}{l}\text { Green, Ender, } \\
\text { \& Ryan (2016) }\end{array}$ & $\begin{array}{l}\text { What is optimal } \\
\text { treatment for } \\
\text { vertiginous } \\
\text { migraine? }\end{array}$ & $\begin{array}{l}\text { Revisão de } \\
\text { literatura }\end{array}$ & $\begin{array}{l}\text { 1-Agonista do receptor 5- } \\
\text { HT1 } \\
\text { 2-Beta-bloqueadores } \\
\text { 3-Bloqueadores dos } \\
\text { canais de cálcio } \\
\text { 4-Antidepressivo inibidor } \\
\text { da recaptação de } \\
\text { serotonina e } \\
\text { noradrenalina } \\
\text { 5-Antagonista dos canais } \\
\text { de cálcio } \\
\text { 6-Vasodilatador } \\
\text { 7-Analgésico }\end{array}$ & $\begin{array}{l}\text { 1-Rizatriptana } \\
\text { 2-Zolmitriptano 2,5mg } \\
\text { 3-Propranolol } 40 \mathrm{a} \\
\text { 160mg } \\
\text { 4-Venlafaxina } 37,5 \mathrm{a} \\
\text { 150mg } \\
\text { 5-Flunarizina 10mg } \\
\text { 6-Betaistina 16mg } \\
\text { 7-Paracetamol }\end{array}$ & $\begin{array}{l}\text { Estudo comparativo entre as } \\
\text { pesquisas realizadas por Furman } \\
\text { et al., Neuhauser et al., Salvis et } \\
\text { al., e Lepcha et al. O estudo } \\
\text { comprovou que não há } \\
\text { evidências suficientes para } \\
\text { definir qual o melhor tratamento } \\
\text { medicamentoso para a MV. No } \\
\text { entanto, o uso de flunarizina } \\
\text { reduziu tanto a frequência } \\
\text { quanto a intensidade das } \\
\text { vertigens na MV. }\end{array}$ \\
\hline $\begin{array}{l}\text { Kalla, Teufel, } \\
\text { Feil, Muth, \& } \\
\text { Strupp (2016) }\end{array}$ & $\begin{array}{l}\text { Update on the } \\
\text { pharmacothe- } \\
\text { rapy of } \\
\text { cerebellar and } \\
\text { central } \\
\text { vestibular } \\
\text { disorders. } \\
\text { Journal of } \\
\text { Neurology } \\
\text { (2016) 263 } \\
\text { (Suppl 1): S24- } \\
\text { S29. }\end{array}$ & $\begin{array}{l}\text { Revisão de } \\
\text { literatura }\end{array}$ & $\begin{array}{l}\text { 1-Bloqueador de canal de } \\
\text { cálcio } \\
\text { 2-Bloqueador de canal de } \\
\text { potássio } \\
\text { 3-Diurético inibidor da } \\
\text { anidrase carbônica } \\
\text { 4-Aminoácido } \\
\text { 5- Bloqueador de canal } \\
\text { de potássio }\end{array}$ & $\begin{array}{l}\text { 1-Metoprolol 95mg/d } \\
\text { (para MV) } \\
\text { 2-Fampridine 20mg } \\
\text { 3-Acetazolamida } \\
\text { 750mg/d (ataxia } \\
\text { episódica tipo 2) } \\
\text { 4-Acetil-DL-leucina } \\
\text { g/d (ataxia cerebelar) } \\
\text { 5-Fampridine 20mg/d } \\
\text { (marcha da ataxia } \\
\text { cerebelar). }\end{array}$ & $\begin{array}{l}\text { Revisão geral sobre testes } \\
\text { clínicos randomizados sobre o } \\
\text { uso de medicamentos para } \\
\text { migrânea vestibular, episódios } \\
\text { de ataxia tipo 2, ataxia cerebelar } \\
\text { e marcha de ataxia cerebelar. } \\
\text { Para a MV, relatou que existem } \\
\text { vários medicamentos para o } \\
\text { tratamento profilático e para as } \\
\text { crises, no entanto não tem } \\
\text { comprovação da eficácia em } \\
\text { testes clínicos randomizados, } \\
\text { exceto o teste PROVEMIG que } \\
\text { ainda está em progresso com o } \\
\text { uso de metoprolol 95mg/d. }\end{array}$ \\
\hline $\begin{array}{l}\text { Çelebisoy et al. } \\
\text { (2016) }\end{array}$ & $\begin{array}{l}\text { Acetazolami-de } \\
\text { in vestibular } \\
\text { migraine } \\
\text { prophylaxis: a } \\
\text { retrospective } \\
\text { study. }\end{array}$ & $\begin{array}{l}\text { Coorte } \\
\text { retrospectivo }\end{array}$ & $\begin{array}{l}\text { Diurético inibidor da } \\
\text { anidrase carbônica }\end{array}$ & Acetazolamida & $\begin{array}{l}\text { Estudo realizado com } 39 \\
\text { pacientes, sendo } 31 \text { mulheres e } 8 \\
\text { homens na idade de } 25 \text { a } 66 \\
\text { anos, todos sem sintomas } \\
\text { neurológicos e otológicos. Neste } \\
\text { estudo, a acetazolamida foi } \\
\text { efetiva na redução da frequência } \\
\text { e intensidade da cefaleia e } \\
\text { vertigem da MV. }\end{array}$ \\
\hline $\begin{array}{l}\text { Barbosa \& } \\
\text { Villa (2016) }\end{array}$ & $\begin{array}{l}\text { Vestibular } \\
\text { migraine: } \\
\text { diagnosis } \\
\text { challenges and } \\
\text { need for targeted } \\
\text { treatment. }\end{array}$ & $\begin{array}{l}\text { Revisão de } \\
\text { literatura }\end{array}$ & $\begin{array}{l}\text { 1-Agonista do receptor 5- } \\
\text { HT1 } \\
\text { 2-Antagonista dos canais } \\
\text { de cálcio } \\
\text { 3-Vasodilatador } \\
\text { 4-Analgésico } \\
\text { 5-Antivertiginoso e } \\
\text { vasodilatador } \\
\text { 6-Anticonvulsivante } \\
\text { 7-Betabloqueador } \\
\text { 8-Betabloqueador } \\
\text { 9-Anticonvulsivante } \\
\text { 10-Anticonvulsivante } \\
\text { 11-Extrato de planta: } \\
\text { chapéu de aba larga } \\
\text { 12-Antidepressivo } \\
\text { tricíclico } \\
\text { 13-Antagonista dos }\end{array}$ & $\begin{array}{l}\text { 1-Zolmitriptana 2,5mg } \\
\text { 2-Flunarizina 10mg } \\
\text { 3-Betaistina } \\
\text { 4-Paracetamol } \\
\text { 5-Cinarizina } \\
\text { 6-Lamotrigina } 75 \mathrm{e} \\
\text { 100mg } \\
\text { 7-Metoprolol 150mg } \\
\text { 8-Propranolol 160mg } \\
\text { 9-Ácido valpróico } \\
\text { 600mg } \\
\text { 10-Topiramato 50mg } \\
\text { 11-Extrato de butterbur } \\
\text { 50mg } \\
\text { 12-Amitriptilina } 75 \mathrm{a} \\
\text { 100mg } \\
\text { 13-Flunarizina 5mg } \\
\text { 14-Magnésio 400mg }\end{array}$ & $\begin{array}{l}\text { Refere que a MV ainda é } \\
\text { subdiagnosticada e que os } \\
\text { estudos sobre o tratamento da } \\
\text { MV são escassos. Comparou } \\
\text { estudos que, em suma, sugerem } \\
\text { que o tratamento profilático da } \\
\text { MV seja baseado em diretrizes } \\
\text { sobre o tratamento da migrânea. } \\
\text { Relata sobre a necessidade de } \\
\text { haver mais estudos controlados } \\
\text { sobre a intervenção } \\
\text { farmacológica no tratamento da } \\
\text { MV. }\end{array}$ \\
\hline
\end{tabular}


Research, Society and Development, v. 10, n. 8, e32110817414, 2021

(CC BY 4.0) | ISSN 2525-3409 | DOI: http://dx.doi.org/10.33448/rsd-v10i8.17414

\begin{tabular}{|c|c|c|c|c|c|}
\hline & & & $\begin{array}{l}\text { canais de cálcio } \\
\text { 14-Eletrólito }\end{array}$ & $\begin{array}{l}\text { 15-Exercício de } \\
\text { reabilitação vestibular. }\end{array}$ & \\
\hline $\begin{array}{l}\text { Ferster, } \\
\text { Priesol, \& } \\
\text { Isildak (2017) }\end{array}$ & $\begin{array}{l}\text { The clinical } \\
\text { manifestations } \\
\text { of vestibular } \\
\text { migraine: a } \\
\text { review. } \\
\text { Auris Nasus } \\
\text { Larynx 44 } \\
\text { (2017) 249-252. }\end{array}$ & $\begin{array}{l}\text { Revisão de } \\
\text { literatura }\end{array}$ & $\begin{array}{l}\text { 1-Betabloqueador } \\
\text { 2- Betabloqueador } \\
\text { 3-Antagonista dos canais } \\
\text { de cálcio } \\
\text { 4-Anti-hipertensivo } \\
\text { 5-Agonista do receptor 5- } \\
\text { HT1 } \\
\text { 6- Antidepressivo } \\
\text { tricíclico } \\
\text { 7-Anticonvulsivante }\end{array}$ & $\begin{array}{l}\text { 1-Propranolol } \\
\text { 2-Bisoprolol } \\
\text { 3-Flunarizina } \\
\text { 4- Metoprolol } \\
\text { 5-Zolmitriptana } \\
\text { 6-Antidepressivo } \\
\text { tricíclico } \\
\text { 7-Gabapentina. }\end{array}$ & $\begin{array}{l}\text { Identificaram as manifestações } \\
\text { clínicas da MV como auras } \\
\text { reversíveis, fotofobia, cefaleia, } \\
\text { vertigem, tontura posicional ou } \\
\text { sensação de desequilíbrio. } \\
\text { Relataram, também, sobre o } \\
\text { diagnóstico e tratamento da MV. } \\
\text { Observaram que os } \\
\text { medicamentos utilizados como } \\
\text { profilaxia dos sintomas da MV } \\
\text { foram propranolol, bisoprolol, } \\
\text { flunarizina, metoprolol e } \\
\text { zolmitriptana, antidepressivo } \\
\text { tricíclico e gabapentina. }\end{array}$ \\
\hline $\begin{array}{l}\text { Liu, Renk, } \\
\text { Rauch, \& Xu, } \\
(2017)\end{array}$ & $\begin{array}{l}\text { Efficacy of } \\
\text { intratympanic } \\
\text { gentamicin in } \\
\text { Menière's } \\
\text { disease with and } \\
\text { without } \\
\text { migraine. } \\
\text { Otology \& } \\
\text { Neurotology } 38 \text { : } \\
\text { 1005-1009, } \\
2017 .\end{array}$ & $\begin{array}{l}\text { Estudo } \\
\text { retrospectivo }\end{array}$ & $\begin{array}{l}\text { Antibiótico } \\
\text { aminoglicosídeo }\end{array}$ & $\begin{array}{l}\text { Sulfato de gentamicina } \\
40 \mathrm{mg} / \mathrm{ml} \text { (antibiótico } \\
\text { injetável } \\
\text { intratimpânico) }\end{array}$ & $\begin{array}{l}\text { Estudo realizado por } 2 \text { anos com } \\
28 \text { pacientes, sendo } 14 \text { no grupo } \\
\text { com migrânea e } 14 \text { no grupo } \\
\text { controle (sem migrânea), do } \\
\text { quais eram } 3 \text { mulheres e } 11 \\
\text { homens, com idade em média de } \\
53 \text { anos. Os autores sugerem que } \\
\text { os pacientes diagnosticados com } \\
\text { MV utilizem a injeção com } \\
\text { gentamicina somente após o } \\
\text { tratamento medicamentoso } \\
\text { convencional, devendo } \\
\text { esclarecer que a melhora } \\
\text { funcional não é pronunciada, } \\
\text { embora diminua os episódios de } \\
\text { vertigem e as crises de migrânea. }\end{array}$ \\
\hline $\begin{array}{l}\text { Salmito et al. } \\
\text { (2017) }\end{array}$ & $\begin{array}{l}\text { Prophylactic } \\
\text { treatment of } \\
\text { vestibular } \\
\text { migraine. } \\
\text { Brazilian Journal } \\
\text { of otorhinolaryn- } \\
\text { gology, 2017; } \\
\text { 83: 404-410. }\end{array}$ & $\begin{array}{l}\text { Estudo } \\
\text { observacional, } \\
\text { longitudinal e } \\
\text { retrospectivo }\end{array}$ & $\begin{array}{l}\text { 1-Antidepressivo } \\
\text { tricíclico } \\
\text { 2-Antagonista dos canais } \\
\text { de cálcio } \\
\text { 3-Antidepressivo } \\
\text { tricíclico } \\
\text { 4-Betabloqueador } \\
\text { 5-Anticonvulsivante } \\
\text { 6-Anticonvulsivante } \\
\text { 7-Antidepressivo inibidor } \\
\text { da recaptação de } \\
\text { serotonina e } \\
\text { noradrenalina }\end{array}$ & $\begin{array}{l}\text { 1-Amitriptilina } 25 \mathrm{mg} \text { e } \\
\text { 50mg } \\
\text { 2-Flunarizina } 10 \mathrm{mg} \\
\text { 3-Nortriptilina } 50 \mathrm{mg} \\
\text { 4-Propranolol } 40 \mathrm{mg} \mathrm{e} \\
80 \mathrm{mg} \\
\text { 5-Topiramato } 100 \mathrm{mg} \mathrm{e} \\
\text { 200mg } \\
\text { 6-Valproato 500mg e } \\
\text { 1000mg } \\
\text { 7-Venlafaxina } 75 \mathrm{mg}\end{array}$ & $\begin{array}{l}\text { Estudo realizado com } 47 \\
\text { prontuários de pacientes com } \\
\text { diagnóstico de MV, com idade } \\
\text { variando de } 19 \text { a } 69 \text { anos. O } \\
\text { estudo mostrou melhora dos } \\
\text { sintomas com o uso profilático } \\
\text { de amitriptilina, flunarizina, } \\
\text { propranolol e topiramato. }\end{array}$ \\
\hline Lapira (2019) & $\begin{array}{l}\text { Vestibular } \\
\text { migraine } \\
\text { treatment and } \\
\text { prevention. } \\
\text { HNO, 2019; 67: } \\
\text { 425-428. }\end{array}$ & $\begin{array}{l}\text { Revisão de } \\
\text { literatura }\end{array}$ & $\begin{array}{l}\text { 1-Betabloqueador } \\
\text { 2-Betabloqueador } \\
\text { 3-Anticonvulsivante } \\
\text { 4-Antidepressivo } \\
\text { 5-Antagonista dos canais } \\
\text { de cálcio }\end{array}$ & $\begin{array}{l}\text { 1-Propranolol 40- } \\
\text { 240mg } \\
\text { 2-Metoprolol 50- } \\
\text { 200mg } \\
\text { 3-Topiramato 50- } \\
\text { 100mg } \\
\text { 4-Amitriptilina 25- } \\
\text { 75mg e 75-150mg } \\
\text { 5-Flunarizina 5-10mg. }\end{array}$ & $\begin{array}{l}\text { Consiste em uso de } \\
\text { medicamentos antidrepressivos, } \\
\text { betabloqueadores, bem como } \\
\text { modificação da dieta e de } \\
\text { comportamento. Para frequentes } \\
\text { episódios vestibulares, necessita } \\
\text { o uso de topiramato como } \\
\text { profilaxia, enquanto que a } \\
\text { amitriptilina deve ser usada para } \\
\text { pacientes ansiosos e com } \\
\text { insônia. }\end{array}$ \\
\hline $\begin{array}{l}\text { Kaya et al. } \\
\text { (2019) }\end{array}$ & $\begin{array}{l}\text { Can verapamil } \\
\text { be effective in } \\
\text { controlling } \\
\text { vertigo and } \\
\text { headache attacks } \\
\text { in vestibular } \\
\text { migraine }\end{array}$ & $\begin{array}{l}\text { Estudo } \\
\text { observacional } \\
\text { retrospectivo }\end{array}$ & $\begin{array}{l}\text { Bloqueador de canal de } \\
\text { cálcio }\end{array}$ & $\begin{array}{l}\text { Verapamil } 80 \mathrm{mg} / \mathrm{dia} \text {, } \\
\text { divididas em duas } \\
\text { doses por } 3 \text { meses. }\end{array}$ & $\begin{array}{l}\text { Estudo realizado com } 17 \\
\text { pacientes de } 28 \text { a } 56 \text { anos, no } \\
\text { período de outubro de } 2015 \text { a } \\
\text { abril de } 2017 \text {. Confirmaram a } \\
\text { eficácia da dose mínima } \\
\text { indicada de verapamil na } \\
\text { redução das vertigens e dores de }\end{array}$ \\
\hline
\end{tabular}




\begin{tabular}{|c|c|c|c|c|c|}
\hline & $\begin{array}{l}\text { accompanied } \\
\text { with Menière's } \\
\text { disease? } \\
\text { Journal of } \\
\text { Neurology } \\
\text { (2019) 266 } \\
\text { (Suppl 1): 562- } \\
564\end{array}$ & & & & $\begin{array}{l}\text { cabeça dos pacientes portadores } \\
\text { de MV e da doença de Menière. } \\
\text { No entanto, sugeriram sobre a } \\
\text { necessidade de haver estudos } \\
\text { randomizados e controlados para } \\
\text { comprovar a eficácia do } \\
\text { verapamil. }\end{array}$ \\
\hline $\begin{array}{l}\text { Çelik, Toker, } \\
\text { Eskiizmir, } \\
\text { Incesulu, \& } \\
\text { Süyür (2020) }\end{array}$ & $\begin{array}{l}\text { The } \\
\text { effectiveness of } \\
\text { medical } \\
\text { prophylactic } \\
\text { treatment on } \\
\text { vestibular } \\
\text { migraine and its } \\
\text { effect on the } \\
\text { quality of life. } \\
\text { The Journal of } \\
\text { Internacional } \\
\text { Advanced } \\
\text { Otology 2020; } \\
16(1): 28-33 \text {. }\end{array}$ & $\begin{array}{l}\text { Estudo } \\
\text { observacional } \\
\text { retrospectivo }\end{array}$ & $\begin{array}{l}\text { Bloqueador de canal de } \\
\text { cálcio }\end{array}$ & $\begin{array}{l}\text { Propranolol 40mg/d, } \\
80 \mathrm{mg} / \mathrm{d} \text { e } 120 \mathrm{mg} / \mathrm{d} \text {. }\end{array}$ & $\begin{array}{l}\text { Estudo realizado com } 38 \\
\text { pacientes diagnosticados com } \\
\text { MV no período janeiro de } 2015 \\
\text { a setembro de } 2017 \text {. A faixa } \\
\text { etária variava de } 18 \text { a } 75 \text { anos. } \\
\text { Todos foram acompanhados por } \\
6 \text { a } 36 \text { meses. Este estudo } \\
\text { mostrou que a duração, a } \\
\text { intensidade e a frequência das } \\
\text { crises foram reduzidas, } \\
\text { aumentando a qualidade de vida } \\
\text { dos pacientes. }\end{array}$ \\
\hline $\begin{array}{l}\text { Sarna, } \\
\text { Abouzari, Lin, } \\
\text { \& Djalilian } \\
(2020)\end{array}$ & $\begin{array}{l}\text { A hypothetical } \\
\text { proposal for } \\
\text { association } \\
\text { between } \\
\text { migraine and } \\
\text { Menière's } \\
\text { disease. } \\
\text { Medical } \\
\text { hypotheses 134, } \\
\text { 2020. Journal } \\
\text { homepage: } \\
\text { www.elsevier.co } \\
\text { m/locate/mehy }\end{array}$ & $\begin{array}{l}\text { Revisão de } \\
\text { literatura }\end{array}$ & $\begin{array}{l}\text { 1-Anti-hipertensivo } \\
\text { 2-Diurético tiazídico } \\
\text { 3-Antidepressivo } \\
\text { tricíclico } \\
\text { 4-Anticonvulsivante }\end{array}$ & $\begin{array}{l}\text { 1-Inibidores da } \\
\text { anidrase carbônica } \\
\text { 2-Hidroclorotiazida } \\
\text { 3-Nortriptilina } \\
\text { 4-Topiramato }\end{array}$ & $\begin{array}{l}\text { Referem que o tratamento } \\
\text { medicamentoso e dietético } \\
\text { utilizados para a migrânea, } \\
\text { também podem ser indicados } \\
\text { para a doença de Menière e para } \\
\text { a migrânea vestibular, por } \\
\text { apresentar sintomas parecidos } \\
\text { com os da doença de Menière. }\end{array}$ \\
\hline $\begin{array}{l}\text { Domínguez- } \\
\text { Durán et al. } \\
\text { (2020) }\end{array}$ & $\begin{array}{l}\text { Analysis of the } \\
\text { effectiveness of } \\
\text { the prophylaxis } \\
\text { of vestibular } \\
\text { migraine } \\
\text { depending on } \\
\text { the diagnostic } \\
\text { category and the } \\
\text { prescribed drug. } \\
\text { European } \\
\text { Archives of Oto- } \\
\text { Rhino- } \\
\text { Laryngology, } \\
2020,277: 1013- \\
1021 .\end{array}$ & $\begin{array}{l}\text { Estudo } \\
\text { prospectivo } \\
\text { multicêntrico }\end{array}$ & $\begin{array}{l}\text { 1- Diurético inibidor da } \\
\text { anidrase carbônica } \\
\text { 2-Antidepressivo } \\
\text { 3-Antagonista dos canais } \\
\text { de cálcio } \\
\text { 4-Bloqueador de canal de } \\
\text { cálcio } \\
\text { 5-Anticonvulsivante }\end{array}$ & $\begin{array}{l}\text { 1-Acetazolamida } \\
\text { 250mg } \\
\text { 2-Amitriptilina 10mg } \\
\text { 3-Flunarizina 5mg } \\
\text { 4-Propranolol 10mg } \\
\text { 5-Topiramato } 25 \mathrm{mg}\end{array}$ & $\begin{array}{l}\text { Pesquisa realizada com } 31 \\
\text { pacientes com provável MV e } \\
\text { com MV, com idade média de } \\
46 \text { anos e cerca de } 71,9 \% \text { eram } \\
\text { mulheres. Mostrou que não há } \\
\text { diferença significante entre as } \\
\text { medicações usadas para } \\
\text { profilaxia da MV, no entanto } \\
\text { sugere que as medicações sejam } \\
\text { adequadas de acordo com as } \\
\text { comorbidades ou hábitos de vida } \\
\text { de cada paciente, conforme } \\
\text { experiência médica. As } \\
\text { medicações testadas nesse } \\
\text { estudo tiveram uma média } \\
\text { redução nos sintomas } \\
\text { vestibulares, das cefaleias } \\
\text { migranosas e nas crises mensais. }\end{array}$ \\
\hline $\begin{array}{l}\text { Liu, Macias, } \\
\text { Donaldson, } \\
\text { Dornhoffer, \& } \\
\text { Rizk (2020) }\end{array}$ & $\begin{array}{l}\text { Pharmaco- } \\
\text { therapy failure } \\
\text { and progression } \\
\text { to botulinum } \\
\text { toxin injection in } \\
\text { vestibular } \\
\text { migraine. } \\
\text { J Laryngol Otol } \\
\text { 2020; } 1-6 .\end{array}$ & $\begin{array}{l}\text { Coorte } \\
\text { retrospectivo }\end{array}$ & $\begin{array}{l}\text { 1-Antidepressivo } \\
\text { tricíclico } \\
\text { 2-Antidepressivo inibidor } \\
\text { da recaptação de } \\
\text { serotonina e } \\
\text { noradrenalina } \\
\text { 3- Anticonvulsivante } \\
\text { 4- Bloqueador de canal } \\
\text { de cálcio } \\
\text { 5- Bloqueador de canal } \\
\text { de cálcio }\end{array}$ & $\begin{array}{l}\text { 1-Nortriptilina } \\
\text { 2-Venlafaxina } \\
\text { 3-Topiramato } \\
\text { 4-Propranolol } \\
\text { 5-Verapamil } \\
\text { 6-Toxina botulínica } \\
\text { 7-Reabilitação } \\
\text { vestibular }\end{array}$ & $\begin{array}{l}\text { Estudo realizado com } 47 \\
\text { pacientes com MV de setembro } \\
\text { de } 2015 \text { a julho de } 2019 \text {. Os } \\
\text { fatores que influenciaram a falha } \\
\text { da terapia medicamentosa e a } \\
\text { necessidade da administração } \\
\text { precoce da toxina botulínica } \\
\text { foram: oscilopsia, falha na } \\
\text { primeira medicação, } \\
\text { hipersensibilidade emocional e o } \\
\text { medo do estigma social. } \\
\text { Argumentam que se o paciente } \\
\text { apresentar esses fatores podem }\end{array}$ \\
\hline
\end{tabular}




\begin{tabular}{|c|c|c|c|c|c|}
\hline & & & $\begin{array}{l}\text { 6-Inibidor da liberação de } \\
\text { acetilcolina }\end{array}$ & & $\begin{array}{l}\text { progredir para o uso da toxina ao } \\
\text { invés de tentar outra medicação. }\end{array}$ \\
\hline $\begin{array}{l}\text { Dornhoffer et } \\
\text { al. }(2021)\end{array}$ & $\begin{array}{l}\text { Factors } \\
\text { implicated in } \\
\text { response to } \\
\text { treatment/ } \\
\text { prognosis of } \\
\text { vestibular } \\
\text { migraine. } \\
\text { European } \\
\text { Archives of Oto- } \\
\text { Rhino- } \\
\text { Laryngology, } \\
\text { 2021, 278: 57- } \\
66 .\end{array}$ & $\begin{array}{l}\text { Coorte } \\
\text { retrospectivo }\end{array}$ & $\begin{array}{l}\text { 1-Antidepressivo inibidor } \\
\text { da recaptação de } \\
\text { serotonina e } \\
\text { noradrenalina } \\
\text { 2-Antidepressivo } \\
\text { tricíclico } \\
\text { 3-Anticonvulsivante } \\
\text { 4-Bloqueador de canal de } \\
\text { cálcio } \\
\text { 5-Bloqueador de canal de } \\
\text { cálcio }\end{array}$ & $\begin{array}{l}\text { 1-Venlafaxina } 37,5 \text { a } \\
75 \mathrm{mg} \\
\text { 2-Nortriptilina } 20 \mathrm{a} \\
\text { 50mg } \\
\text { 3-Topiramato } 25 \mathrm{a} \\
\text { 50mg } \\
\text { 4-Propranolol 80mg 5- } \\
\text { Verapamil 120mg 6- } \\
\text { Mudanças de estilo de } \\
\text { vida (atividade física e } \\
\text { dieta) }\end{array}$ & $\begin{array}{l}\text { Estudo com } 47 \text { pacientes } \\
\text { diagnosticados com MV entre } \\
\text { setembro de } 2015 \text { a junho de } \\
2019 \text {. Os pacientes foram } \\
\text { submetidos ao protocolo de } \\
\text { tratamento com antidepressivos, } \\
\text { antiepilépticos, } \\
\text { betabloqueadores e reabilitação } \\
\text { vestibular. Os pacientes que não } \\
\text { tiveram sucesso com o uso } \\
\text { concomitante de } 3 \text { classes de } \\
\text { medicamentos foram indicados } \\
\text { para uso da toxina botulínica. Os } \\
\text { fatores que mais influenciaram } \\
\text { na diminuição dos sintomas } \\
\text { foram: gênero feminino, } \\
\text { presença de vertigem posicional } \\
\text { paroxística benigna e um nível } \\
\text { elevado de tontura. Outros } \\
\text { aspectos como cervicalgia e } \\
\text { oscilopsia tiveram um resultado } \\
\text { mais baixo no Dizziness } \\
\text { Handicap Inventory (DHI). O } \\
\text { reconhecimento da influência } \\
\text { desses fatores é importante para } \\
\text { direcionar os médicos no } \\
\text { tratamento e prognóstico de seus } \\
\text { pacientes. }\end{array}$ \\
\hline
\end{tabular}

Fonte: Autores.

Entre os 14 trabalhos analisados, observou-se que apenas 4 (28\%) realizaram o tratamento da MV com apenas 1 medicamento: acetazolamida, sulfato de gentamicina, verapamil e propranolol, sendo respectivamente das classes diurético inibidor da anidrase carbônica, aminoglicosídeo, bloqueador dos canais de cálcio e betabloqueador, todos evidenciaram melhora dos sintomas (Çelebisoy et al., 2016; Liu et al., 2017; Kaya et al., 2019; Çelik et al., 2020).

Em relação às classes medicamentosas relatadas nos trabalhos, 7 (50\%) utilizaram tratamento com antidepressivos, betabloqueadores e anticonvulsivantes concomitantemente (Barbosa \& Villa, 2016; Ferster et al., 2017; Salmito et al., 2017; Lapira, 2019; Domínguez-Durán et al., 2020; Liu et al., 2020; Dornhoffer et al., 2021).

Em apenas $4(28 \%)$ trabalhos, a mudança do estilo de vida foi considerada como terapêutica concomitante fundamental para o sucesso do tratamento (Lapira, 2019; Çelik et al., 2020; Sarna et al, 2020; Dornhoffer et al., 2021).

O uso da toxina botulínica foi encontrado em apenas 2 (14\%) artigos analisados, sendo utilizada quando não há sucesso no primeiro tratamento medicamentoso ou no uso de três medicamentos concomitantes (Liu et al., 2020; Dornhoffer et al., 2021).

$\mathrm{O}$ uso da injeção com gentamicina em pacientes diagnosticados, simultaneamente, com doença de Menière e VM foi relatado em 1 (7\%) trabalho e seu uso só deverá ser realizado após o tratamento medicamentoso convencional da MV, podendo ter resultados como a redução das quedas repentinas e dos episódios de vertigem (Liu et al., 2017).

Todos os 14 (100\%) trabalhos relataram que há uma necessidade de mais estudos randomizados e controlados acerca do tratamento medicamentoso da migrânea vestibular, com o intuito de se estabelecer fármacos específicos para esta patologia.

\section{Discussão}

Corroborando com todos os autores dos 14 trabalhos analisados, os pacientes foram incluídos no diagnóstico de migrânea vestibular, baseado nos critérios diagnósticos da terceira edição da Classificação Internacional de Cefaleias (Lempert, 2013). 
O tratamento da migrânea vestibular pode ser realizado de forma profilática e durante os períodos de crise, podendo ser prescritos classes de medicamentos como: betabloqueadores, antidepressivos e anticonvulsivantes. Em estudo realizado em prontuários de 47 pacientes com diagnóstico de MV, mostrou melhora significante dos sintomas com o uso profilático de amitriptilina, flunarizina, propranolol e topiramato (Salmito et al., 2017).

Os antidepressivos são utilizados como medicamento profilático das crises de MV, por apresentar melhora representativa dos sintomas. Deve ser iniciado de forma gradual e após o controle dos sintomas, deve ser mantido por no mínimo 6 meses. Se houver a necessidade de retirar o medicamento, esta deve ser de forma gradual com o objetivo de evitar sintomas de abstinência. Na profilaxia das crises de migrânea vestibular, utilizam-se nortriptilina e amitriptilina e antidepressivos que agem inibindo a recaptação de noradrenalina e serotonina. Foi observado que os antidepressivos foram utilizados em 9 (64\%) dos trabalhos analisados (Green et al., 2016; Barbosa \& Villa, 2016; Ferster et al., 2017; Salmito et al., 2017; Lapira, 2019; Sarna et al., 2020; Dominguez-Durán et al., 2020; Liu et al., 2020; Dornhoffer et al., 2021).

Os anticonvulsivantes mais utilizados no tratamento da MV foram topiramato, valproato, gabapentina e lamotrigina, sendo evidenciado nos trabalhos avaliados (Barbosa \& Villa, 2016; Ferster et al., 2017; Salmito et al., 2017; Lapira, 2019; Sarna et al., 2020; Dominguez-Durán et al., 2020; Liu et al., 2020; Dornhoffer et al., 2021).

No tratamento profilático da migrânea vestibular, o uso de propranolol teve efeitos positivos quanto à redução da intensidade, frequência e número de ataques, proporcionando uma melhor qualidade de vida, sendo observado em 9 (64\%) trabalhos analisados (Green et al., 2016; Barbosa \& Villa, 2016; Ferster et al., 2017; Salmito et al., 2017; Lapira, 2019; Çelik et al., 2020; Dominguez-Durán et al., 2020; Liu et al., 2020; Dornhoffer et al., 2021). Enquanto que em um dos trabalhos avaliados, foi relatado que apesar de não haver evidências suficientes para definir qual o tratamento ideal da MV, foi sugerido que o uso de flunarizina reduziu a frequência e a intensidade das vertigens na MV (Green et al., 2016).

Em 2017, o estudo realizado por Ferster et al. (2017) indicou os medicamentos utilizados como profilaxia dos sintomas da MV foram propranolol, bisoprolol, flunarizina, metoprolol e zolmitriptana, antidepressivo tricíclico e gabapentina. Enquanto que para Dominguez-Durán et al. (2020), não houve diferença significativa entre as medicações utilizadas para a profilaxia da MV, pois todas tiveram uma redução dos sintomas vestibulares e das cefaleias migranosas.

Apesar de haver fatores que provocam uma crise de MV como menstruação, estresse, insônia, desidratação, bem como alguns alimentos, por não terem sido bem estudadas nem a especificidade nem a sensibilidade, tem poucas evidências científicas. Os pacientes que utilizaram doses diárias de amitriptilina $25 \mathrm{mg}$, flunarizina $10 \mathrm{mg}$, propranolol $80 \mathrm{mg}$ e topiramato $100 \mathrm{mg}$ apresentaram melhoria da cefaleia e dos sintomas vestibulares. O propranolol parece necessitar de $80 \mathrm{mg} / \mathrm{dia}$ para apresentar melhora no quadro clínico do paciente (Salmito et al., 2017). No entanto, é importante que o paciente além de usar antidepressivos e betabloqueadores, faça mudança em sua dieta e em seu estilo de vida (Lapira, 2019).

Os medicamentos utilizados para tratamento profilático da migrânea vestibular parecem melhorar os sintomas dessa doença. No entanto, a única vantagem em se utilizar antidepressivos em pacientes com diagnóstico de depressão ou antihipertensivos para aqueles com diagnóstico de hipertensão arterial sistêmica (HAS) é o aprimoramento da farmacoterapia (Salmito et al., 2017), mas se houver episódios vestibulares frequentes, deve-se prescrever topiramato como profilaxia, enquanto que a amitriptilina deve ser usada para pacientes ansiosos e com insônia (Lapira, 2019).

Segundo Salmito et al. (2017), a dificuldade de escolher a melhor opção para os diferentes pacientes com MV permanece, devendo-se basear esta escolha de acordo com contraindicações e interações medicamentosas. Nota-se, assim, a importância do estudo sobre os medicamentos que são utilizados tanto na profilaxia quanto durante a crise da MV, proporcionando ao paciente um cuidado de forma integral, com a finalidade de prevenir e controlar as crises, adequando o tratamento ideal para cada paciente de acordo com sua especificidade, comorbidades e sintomas. 


\section{Conclusão}

Foi possível identificar e determinar as classes medicamentosas e os fármacos mais utilizados na profilaxia e nos períodos de crise da migrânea vestibular, como os betabloqueadores, os anticonvulsivantes, os antidepressivos e antagonistas dos canais de cálcio. É de fundamental importância que o tratamento medicamentoso seja feito sempre associado à mudança do estilo de vida e de forma profilática das crises de MV, para que haja um impacto positivo na vida diária e no bem-estar dos pacientes.

A partir da realização da análise dos artigos sobre o tratamento da migrânea vestibular, obteve-se um conhecimento aprofundado desta patologia, no que se refere às classes e aos medicamentos utilizados para seu tratamento, proporcionando um manejo mais adequado dos fármacos utilizados para combater os sintomas da MV.

Em virtude da importância da temática avaliada, sugere-se que novas pesquisas sobre a intervenção farmacológica no tratamento da migrânea vestibular sejam desenvolvidas, favorecendo expansão técnico-científica, ações de políticas públicas e, ainda, lançar base para uma possível padronização do tratamento específico para MV, viabilizando uma melhor qualidade de vida da população acometida por esta patologia.

\section{Referências}

Barbosa, F., \& Villa, T. R. (2016). Vestibular migraine: Diagnosis challenges and need for targeted treatment. In Arquivos de Neuro-Psiquiatria, 74(5), 416422. Associação Arquivos de Neuro-Psiquiatria. https://doi.org/10.1590/0004-282X20160037

Beh, S. C. (2019). Vestibular Migraine: How to sort it out and what to do about it. Journal of Neuro-Ophthalmology, 39(2), 208-219. https://doi.org/10.1097/WNO.0000000000000791

Çelebisoy, N., Gökçay, F., Karahan, C., Bilgen, C., Kirazlı, T., Karapolat, H., \& Köse, T. (2016). Acetazolamide in vestibular migraine prophylaxis: a retrospective study. European Archives of Oto-Rhino-Laryngology, 273(10), 2947-2951. https://doi.org/10.1007/s00405-015-3874-4

Çelik, O., Toker, G. T., Eskiizmir, G., İncesulu, A., \& Süyür, N. S. (2020). The effectiveness of medical prophylactic treatment on vestibular migraine and its effect on the quality of life. Journal of International Advanced Otology, 16(1), 28-33. https://doi.org/10.5152/iao.2019.6522

Domínguez-Durán, E., Montilla-Ibáñez, M. A., Álvarez-Morujo de Sande, M. G., Domènech-Vadillo, E., Bécares-Martínez, C., González-Aguado, R., \& Guerra-Jiménez, G. (2020). Analysis of the effectiveness of the prophylaxis of vestibular migraine depending on the diagnostic category and the prescribed drug. European Archives of Oto-Rhino-Laryngology, 277(4), 1013-1021. https://doi.org/10.1007/s00405-020-05802-5

Dornhoffer, J. R., Liu, Y. F., Donaldson, L., \& Rizk, H. G. (2021). Factors implicated in response to treatment/prognosis of vestibular migraine. European Archives of Oto-Rhino-Laryngology, 278(1), 57-66. https://doi.org/10.1007/s00405-020-06061-0

Ercole, F. F., Melo, L. D., \& Alcoforado, C. L. G. C. (2014). Integrative review versus systematic review. Rev Min Enferm, 18(1), 9-12. http://www.dx.doi.org/10.5935/1415-2762.20140001

Green, K., Ender, L., \& Winters, R. (2016). What is optimal treatment for vertiginous migraine? In Laryngoscope, 126(12), 2647-2648. John Wiley and Sons Inc. https://doi.org/10.1002/lary.26092

Huang, T. C., Wang, S. J., \& Kheradmand, A. (2020). Vestibular migraine: An update on current understanding and future directions. Cephalalgia, 40(1), 107-121. https://doi.org/10.1177/0333102419869317

Kalla, R., Teufel, J., Feil, K., Muth, C., \& Strupp, M. (2016). Update on the pharmacotherapy of cerebellar and central vestibular disorders. In Journal of Neurology, 263, 24-29. Dr. Dietrich Steinkopff Verlag GmbH and Co. KG. https://doi.org/10.1007/s00415-015-7987-X

Kaya, I., Eraslan, S., Tarhan, C., Bilgen, C., Kirazli, T., Gokcay, F., Karapolat, H., \& Celebisoy, N. (2019). Can verapamil be effective in controlling vertigo and headache attacks in vestibular migraine accompanied with Menière's disease? A preliminary study. In Journal of Neurology, 266, 62-64. Dr. Dietrich Steinkopff Verlag GmbH and Co. KG. https://doi.org/10.1007/s00415-019-09309-w

Lapira, A. (2019). Vestibular migraine treatment and prevention. In HNO. Springer Verlag. https://doi.org/10.1007/s00106-019-0661-3

Lempert, T. (2013). Vestibular migraine. Seminars in Neurology, 33(3), 212-218. https://doi.org/10.1055/s-0033-1354596

Liu, Y. F., Macias, D., Donaldson, L., Dornhoffer, J. R., \& Rizk, H. G. (2020). Pharmacotherapy failure and progression to botulinum toxin injection in vestibular migraine. Journal of Laryngology and Otology, 134(7), 586-591. https://doi.org/10.1017/S002221512000095X

Liu, Yuan F., Renk, E., Rauch, S. D., \& Xu, H. X. (2017). Efficacy of intratympanic gentamicin in Menière's disease with and without migraine. Otology and Neurotology, 38(7), 1005-1009. https://doi.org/10.1097/MAO.0000000000001460

Moher, D., Liberati, A., Tetzlaff, J., \& Altman, D. G. (2009). Preferred reporting items for systematic reviews and meta-analyses: The PRISMA Statement. PLoS Medicine, 6(7), e1000097. https://doi.org/10.1371/journal.pmed.1000097 
Research, Society and Development, v. 10, n. 8, e32110817414, 2021

(CC BY 4.0) | ISSN 2525-3409 | DOI: http://dx.doi.org/10.33448/rsd-v10i8.17414

Morganti, L. O. G., Salmito, M. C., Duarte, J. A., Bezerra, K. C., Simões, J. C., \& Ganança, F. F. (2016). Migrânea vestibular: aspectos clínicos e epidemiológicos. Brazilian Journal of Otorhinolaryngology, 82(4), 397-402. https://doi.org/10.1016/j.bjorl.2015.06.003

Ferster, A. P., Priesol, A. J., \& Isildak, H. (2017). The clinical manifestations of vestibular migraine: A review. In Auris Nasus Larynx, 44(3), 249-252. Elsevier Ireland Ltd. https://doi.org/10.1016/j.anl.2017.01.014

Salmito, M. C., Duarte, J. A., Morganti, L. O. G., Brandão, P. V. C., Nakao, B. H., Villa, T. R., \& Ganança, F. F. (2017). Tratamento profilático da migrânea vestibular. Brazilian Journal of Otorhinolaryngology, 83(4), 404-410. https://doi.org/10.1016/j.bjorl.2016.04.022

Salmito, M. C., e Maia, F. C. Z., Gretes, M. E., Venosa, A., Ganança, F. F., Ganança, M. M., Mezzalira, R., Bittar, R. S. M., Gasperin, A. C., Pires, A. P. B. de Á., Ramos, B. F., Bertoldo, C., Ferreira, C., Real, D., Guimarães, H. A., Oiticica, J., Lavinsky, J., Lopes, K. C., Duarte, J. A., \& Carvalho, R. C. B. de. (2020). Neurotology: definitions and evidence-based therapies - Results of the I Brazilian Forum of Neurotology. Brazilian Journal of Otorhinolaryngology, 86(2), 139-148. https://doi.org/10.1016/j.bjorl.2019.11.002

Sarna, B., Abouzari, M., Lin, H. W., \& Djalilian, H. R. (2020). A hypothetical proposal for association between migraine and Menière's disease. Medical Hypotheses, 134. https://doi.org/10.1016/j.mehy.2019.109430 